\title{
Agent-based Architecture for Flexible Lean Cell Design, Analysis and Evaluation
}

\author{
T.E. Potok, N.D. Ivezic, N.F. Samatova \\ Collaborative Technologies Research Center, Computer Science and Mathematics Division, \\ Oak Ridge National Laboratory, Oak Ridge,TN, USA \\ E-mail:potokte@ornl.gov
}

Keywords supply chain information infrastructure, lean cell formation, agent-based systems

\begin{abstract}
We present an agent architecture in support of lean operations across manufacturing supply chains. Our architecture addresses the issue of heterogeneous information management, the need for analyzing mixed manufacturing approaches, and efficiency and accuracy tradeoffs of analysis methods. We propose five distinct layers to address these requirements: an XML/RDF/DAML Information Access Layer, a Resource Vector Representation Layer, an Analysis Layer, a Coordination Layer, and a Visualization Layer. Our successful results in developing agent systems in support of lean manufacturing cell and supply chain management validate our layered approach.
\end{abstract}

\section{INTRODUCTION}

In today's manufacturing environment, the concept of producing the right product, at the right time, for the right price is a driving goal. To achieve this goal, a manufacturer must have great insight into the supply chain that feeds the manufacturing process. Many would advocate the use of MRP or ERP software systems to achieve this objective. The drawbacks with this approach range from an enormous initial investment to regimenting dated practices. Once such a system is put into place, it can stifle the move to new manufacturing technologies. We believe that the flexibility of agent-based systems provides a great benefit over existing manufacturing control software.

We propose a flexible agent framework that is based on rich representation of manufacturing resources, and advanced analysis 
techniques. With this framework, we can address two key problems within the manufacturing supply chain. The first being the formation of lean or efficient manufacturing cells, and the second is the analysis of material and part flows through the supply chain. The first problem looks at optimal ways of organizing a manufacturing environment, or multiple environments. The second looks at how to understand and analyze the flow of activities that are required to build products.

We have recently developed and deployed two agent-based systems for optimizing manufacturing processes and analyzing supply chain logistics [13] that provide great insight into these problems. From our experience with building agent-based systems for supply chain analysis, we believe that agent technology is ideally suited to be the foundation for a supply chain information infrastructure. In this paper we present an adaptive framework and some preliminary findings for such systems.

\section{BACKGROUND}

The idea of software agents interacting with a manufacturing environment is not a new one. The typical approach reported in the literature is to have agents represent various components of manufacturing, such as parts, machines, cells, and people [4]. Through negotiations among these agents, work can be efficiently scheduled based on a variety of possible constraints. However, the scheduling of work is not performed from a systematic engineering approach, but from efficiencies gained from advanced negotiations techniques [5]. These negotiation-based techniques have proven very effective. However, rather than generalizing these concepts over the supply chain, we believe there may be a more effective approach. Rather than letting agents simulate real-world human interactions, we propose that agents operate based on manufacturing efficiency principles. By doing so, these agents can help build, analyze, optimize manufacturing cells and supply chains from engineering principles. We develop our framework based on two aspects of supply chain analysis, 1) the formation of lean or efficient manufacturing cells, and 2) the analysis of material and part flows through the supply chain.

\section{AGENT FRAMEWORK}

Several unique challenges arise when building agent-based systems.

1. The agent framework must be able to access and understand heterogeneous and distributed information within the supply chain system. Typically, each supplier will represent and store information in 
different ways than other suppliers. This information may include metrics on part inventories, machine utilizations, or production goals.

2. The agent framework must be flexible enough to be able to adapt to different manufacturing methods. One supplier may produce products using traditional batch production methods, with a focus on machine utilization, while another supplier may use agile production processes, where a part value stream analysis is important.

3. The agents must be able to adapt to different analysis methods. There are times when a quick, ballpark estimate may be required, and other times when an in-depth analysis is necessary. The system must be capable of producing both types of analysis.

The typical way of addressing these requirements is to employ an MRP or ERP system that integrates a vast amount of information, and makes this information available to a broader range of participants. However, unless the suppliers in the supply chain adopt the same system, the information will be available only from within a single manufacturing environment, and not the entire supply chain. Other approaches, such as distributed databases, or distributed object brokers can provide a solution, however, a great deal of specialty software must be written and maintained.

A software agent approach provides a number of attractive features. One is the flexibility of communication among agents. Given a common framework, agents throughout the supply chain can communicate to determine the best course of action. Another is the distributed nature of agents, where the information can literally be anywhere.

Looking at the above requirements in terms of agent frameworks provides a more complete justification for a supply chain information framework. Much has been written about the first requirement. The challenge of this requirement is quite pervasive, and unfortunately, current technology is not able to provide much help.

In order for an agent to be able to gather and understand manufacturing information, the agent must have a priori knowledge of the information, such as how the information is organized, what it means, how it is formatted, and what units it is in. Our proposal is to build an encapsulation layer from the current and emerging standards: XML, RDF, and DAML. The Extensible Markup Language (XML) is the universal format for structured documents and data on the Web; The Resource Description Framework (RDF) is a foundation for processing metadata; it provides interoperability between applications that exchange machine-understandable information on the Web; DARPA Agent Markup Language (DAML) is an emerging facility built on top of RDF to enable creation of a Semantic Web and fully interoperable agents. 
The second requirement is critical to the manufacturing environment. If the representation of information is limited by the current manufacturing technology, then changing to a new technology will require a dramatic change to the existing information infrastructure as well. The resources of the manufacturing environment must be represented in a way so that improvement in the future will not require changes to the resource representation. This representation must be flexible and capture essential information about a resource, and critical relationships among resources. We believe that a vector-based representation for manufacturing resources would be ideal for the resource description, cell analysis, and visualization.

The third requirement is to be able to direct the level of analysis needed. The general impact of a change for forecasting purposes will require much different analysis than that needed for short-term production planning. This requirement calls for the user to be able to choose between the duration of analysis versus the accuracy of the analysis. Again, a key aspect of this challenge is to represent this information in such a way that various levels and types of analysis can be performed.

The key to our framework is how the supply chain is represented, and how agents are used to analyze this information. We propose five layers as shown in Figure 1:1) an XML/RDF/DAML access layer that provides a means of searching, accessing, and interpreting manufacturing information. 2) a resource vector representation that relates resources, such as parts and machines, so that each process step for a part is represented as a dimension in space. 3) an analysis layer where this part/machine information can be clustered or grouped to form cells, 4) a coordination layer to enhance agent communication; and 5) a visualization layer where the results of this analysis can be displayed.

\begin{tabular}{|c|c|}
\hline \multirow{4}{*}{$\begin{array}{c}\text { Manufacturing } \\
\text { Agents }\end{array}$} & Visualization Layer \\
\cline { 2 - 3 } & Coordination Layer \\
\cline { 2 - 3 } & Analysis Layer \\
\cline { 2 - 3 } & Resaurce Vector Represen tation Layer \\
\cline { 2 - 2 } & XML/RDF/DAML Access Laye \\
\hline & Manufacturing Information \\
\hline
\end{tabular}

Figure 1 The five layer agent framework for supply chain information analysis.

\subsection{XML/RDF/DAML Access Layer}


This layer provides access and definition to the information available from the manufacturing environment. The information can be gathered, converted or transformed to feed into the resource representation layer.

We have used the extended markup language (XML) as a means of describing and integrating distributed information. XML can provide an encapsulation layer that provides the ability to translate and manipulate representations of information. This XML representation can form a basis for an ontology for a broader range of agents to access and understand the information sources. Furthermore, this XML representation can be used as a method to define uniform syntax of a language for communication among agents.

To achieve a full ontological foundation of communicating agents, additional components of the layer are needed. We have experimented with RDF, a simple, but extensible and potentially powerful representational layer delivered on top of XML. In addition, we have begun to investigate the impact of DAML to provide much more expressive semantics on top of the RDF layer using formal logic to express concepts, relationships, rules, and functions. The XML/RDF/DAML progression of increasingly expressive languages allows one to capture the meaning of manufacturing concepts for their use by higher layers that perform manufacturing-specific activities.

\subsection{Resource Vector Representation Layer}

The second layer calls for a solid representational structure for a manufacturing environment. These systems are typically represented by traditional means, such as a bill of material, or a part-machine matrix. These are usually one or two-dimensional structures, and fairly simple to manipulate. Unfortunately, they are not well suited for representing other factors, such as the direction of the flow of a part through a cell, or the similarity of resources. We believe that with a stronger representational scheme, the agents will be able to provide better information and improved analysis.

We propose a representational structure called a Vector Space Model $(V S M)$. This model helps us to capture all of the relevant features of a resource in relationship to other resources. The basic premise in the VSM is that manufacturing resources, such as machines and parts are represented as elements of a multi-dimensional vector space. Specifically, parts and machines are all vectors in the vector space. The mathematical framework is composed of a k-dimensional vector space and standard linear algebra operations on vectors. In general, the use of a vector space model requires the specification of several aspects such as the set of basis vectors, the 
dimensionality of the space, an interpretation of the values for vector coordinates, and a similarity measure or distance between two vectors.

One of the main virtues of the VSM is the ease with which individual vectors can be modified, making it possible to adapt resource vectors to a dynamic environment without reconsidering the entire data set. Moreover, the vector representational structure provides the desirable flexibility to the Analysis and Visualization Layers.

\subsection{Analysis Layer}

Once we have a strong representation of the manufacturing information, we then are able to quickly analyze the information, and make recommendations. There are two key ways of analyzing the VSM representation. The first is by grouping vectors that are similar into clusters. These clusters can be parts that are manufactured in a common way, machine capabilities, or personnel skills. The other way to analyze this information is by looking at the projection of the multidimensional vectors onto two or three dimensions. This provides a meaningful way of viewing information.

The Vector Perturbation Approach [6] integrated into the Analysis Layer is capable of directing the level of analysis needed. Different aspects specific to a manufacturing environment can be emphasized or obscured due to the variations of the vector perturbation parameter. For instance, it can provide a desirable compromise between the ease of resource assignment and the increase in production throughput by taking advantage of interleaving various parts through a common set of sequenced operations.

This method was applied to three large sets of military aircraft part data. The results, though preliminary, are very promising. Our conclusion is that the VSM representational model coupled with the Vector Perturbation Approach are well suited to address the supply chain information analysis problem, and can be used to enhance the efficiency and utilization of most supply chains.

\subsection{Coordination Layer}

The coordination layer supports interactions within multi-agent systems. We have experimented with skeleton-based approaches such as described in [7]. This approach captures constraints on, and specifications for agent behaviour, specifically, within the context of an agent's participation in dialogs with other agents. Such dialogs may arise through coordination with other agents and collaborative problem solving, to name a few. 
The basis for an agent behavior description using skeletons is messages sent or received by the agent. Each message has a corresponding event specified by a skeleton. A skeleton is a directed, possibly cyclic, graph that consists of states (nodes) and events (links). Each graph has a unique start node and one or more end nodes. Each event (link) represents a transition from its start state to its end state. Each state and event has an associated name, action, and type. The actions associated with states and events are executables. The types of states and events are defined based on the semantics of the intended use of skeletons. Temporal logics can be defined over the skeletons to capture time-dependency among events and states in groups of agents.

\subsection{Visualization Layer}

The visualization layer must be very versatile to represent different types of information in an appropriate way for a human user. We have developed several components of this layer. First, we developed a capability to visualize statistical properties of a distribution of resources or their measurements over some manufacturing space. Second, we developed a capability to render results of statistical clustering methods that we used extensively within some part/resource grouping algorithms. Finally, we developed animation component to visualize interactions/communications among agents, movements of materials and products, and the evolution of important manufacturing concepts such as critical path analysis.

\section{SUMMARY}

An agent-based architecture has been proposed for supply chain information analysis based on manufacturing efficiency principles. Such an architecture facilitates heterogeneous and distributed information processing, flexibility, and adaptability. Flexibility is facilitated through a solid representational structure for a manufacturing environment. Adaptation is supported through the layered structure of the architecture and through multi-level analysis mechanisms.

The architecture is built on the information integration and agents communication standards. The proposed architecture also addresses other specific requirements for next generation manufacturing systems, including scalability, interoperability, cooperation, and visualization.

The experimental results from applying the architecture to the formation of lean manufacturing cells, and the analysis of material and part flows through the supply chain have demonstrated the potential of the agent-based 
approach for advanced supply chain analysis systems. The architecture is generic and can be applied to heterogeneous and distributed information analysis systems in other domains in addition to that of supply chains.

\section{REFERENCES}

[1] Potok T.E., Ivezic N.A. (1999). Multi-agent Spare Part Grouping System for Logistics Optimization, Proceedings of the $5^{\text {th }}$ International Conference on Information Systems Analysis and Synthesis, 582-585.

[2] Ivezic N.A., Potok T.E. (1999). An Agent-Based Approach for Supply Chain Management, Proceedings of the $5^{\text {th }}$ International Conference on Information Systems Analysis and Synthesis, 570-573.

[3] Ivezic N.A., Potok T.E., Pouchard L. (1999). Manufacturing Multiagent Framework for Transitional Operations. IEEE Internet Computing, 3 (5), 58-59.

[4] Cantamessa M. (1997). Agent-based modelling and management of manufacturing systems, Computers in Industry, 34, 173-186.

[5] Baker A.F., Parunal H.V.D., Erol K. (1999). Agents and Internet: Infrastructure for Mass Customization, IEEE Internet Computing, 9, 62-69.

[6] Samatova N.F., Potok T.E., Leuze M.R. (2000). A Vector Perturbation Approach to the Generalized Aircraft Spare Parts Grouping Problem, Proceedings of the $10^{\text {th }}$ International Conference on Flexible Automation and Intelligent Manufacturing, 10171026.

[7] Singh M.P. (2000). Synthesizing Coordination Requirements for Heterogeneous Autonomous Agents, Autonomous Agents and Multi-Agent Systems, 3(2), 107-132. 